\title{
Promising best practices that lead to employment in vocational rehabilitation: Findings from a four-state multiple case study
}

\author{
Roy Del Valle ${ }^{\mathrm{a}, *}$, Michael J. Leahy ${ }^{\mathrm{a}}$, Susan Sherman ${ }^{\mathrm{a}}$, Catherine A. Anderson ${ }^{\mathrm{b}}$, Timothy Tansey ${ }^{\mathrm{c}}$ \\ and Barbara Schoen ${ }^{\mathrm{d}}$ \\ ${ }^{a}$ Michigan State University, East Lansing, MI, USA \\ ${ }^{\mathrm{b}}$ University of Wisconsin-Stout, Menomonie, WI, USA \\ ${ }^{\mathrm{c}}$ University of Wisconsin-Madison, Madison, WI, USA \\ ${ }^{\mathrm{d}}$ The University of Texas at El Paso, El Paso, TX, USA
}

Revised/Accepted July 2014

\begin{abstract}
The state-federal vocational rehabilitation (VR) program has been challenged to demonstrate the effectiveness of VR services provided to eligible consumers. State-federal VR agencies are under pressure to develop a foundation of evidence-based practices that lead to competitive employment outcomes for people with disabilities. This article provides a qualitative analysis of the organization structural elements and service delivery practices related to the provision of effective best practices that enhance employment outcomes for individuals with disabilities. Using a Consensual Qualitative Research (CQR) approach, a multi-stage qualitative analysis of four high performing state VR agencies was conducted to identify promising organizational and service delivery practices that facilitate employment outcomes. Twenty-nine practices were identified as being supportive of the successful outcomes of persons with disabilities being served by these agencies. The identified practices reflect unique and similar service patterns across the four VR agencies studied. Practices were divided into Promising Organizational Practices and Promising Service Delivery Practices. The culture of an organization is an important factor in the development and sustainability of innovative practices. Best practices reconsider traditional rehabilitation counselor roles and offer new methods for delivering services. Rigorous research and program evaluation are required to identify which practices will ultimately constitute evidence-based practices.
\end{abstract}

Keywords: Evidence-based, promising practices, service delivery, organizational practices

\section{Introduction}

Budget challenges at the national and state levels and shrinking public funding have budget-minded employers and government agencies asking hard questions about expected outcomes of rehabilitation services, and seeking objective evidence to justify their support of rehabilitation services (Chan, Rosenthal, \&

\footnotetext{
*Address for correspondence: Roy Del Valle, Office of Rehabilitation and Disability Studies, Michigan State University, 455 Erickson Hall, East Lansing, MI 48824, USA. Tel.: +517 974 6280; Fax: +517 353 6393; E-mail: delvalle@msu.edu.
}

Pruett, 2008). The Work Incentives Improvement Act of 1999 and the Workforce Investment Act of 1998 require the state-federal vocational rehabilitation (VR) program to demonstrate service efficacy in order to maintain and expand program funding and services (Koscuilek, 2004). State VR agencies are under pressure to demonstrate the effectiveness of VR services provided to eligible agency consumers that lead to competitive employment outcomes by using evidencebased practices (EBP) or developing and sharing best practices (Patton, 2008). Rehabilitation counselors in public VR programs must demonstrate evidence-based 
interventions in their practice to improve employment outcomes for consumers with significant disabilities (Rubin, Chan, \& Thomas, 2003). The emphasis on demonstrating VR service delivery effectiveness challenges state VR agencies to address the long-standing question posited by Paul (1967), "What treatment, by whom, is most effective for this individual with that specific problem, and under which set of circumstances?" (p. 111).

Although the general effectiveness of vocational rehabilitation counseling has been empirically demonstrated (Pruett, Rosenthal, Swett, Lee, \& Chan, 2008) there is a serious lack of specific evidence-based practices that accurately define what specific VR service(s) produce employment outcomes for individuals with disabilities that participate in the state VR program (Leahy \& Arokiasamy, 2010). In particular, Law (2002) states that current rehabilitation interventions are not empirically supported, but are primarily based on experience, eminence based or habit based. State VR agencies must understand what service provision patterns have a high probability of predicting successful outcomes for VR consumers. As Chan, Taryvdas, Blalock, Strauser, and Atkins (2009) state, "rehabilitation counseling must [begin to] embrace an evidence-based practice paradigm to remain a vital and respected member of the future community of professionals in rehabilitation and mental health care" (p. 114).

Developing EBP has been emphasized by the National Institute on Disability and Rehabilitation Research (NIDRR) in all research projects publicly funded through NIDRR (Brannon, 2010). Leahy, Thielsen, Millington, Austin, and Fleming (2009) state the emphasis in the future will be on the meaning of research findings to practitioners and consumers in improving services and employment outcomes, and translating and disseminating evidence-based practices so they affect and inform practice and policy. NIDRR and the Rehabilitation Services Administration (RSA), as the major funding sources of rehabilitation research, are working to close the gap between practice and EBP. Specifically, RSA is concerned about developing best practices that provide evidence of effectiveness or efficacy of a practice that will potentially increase the likelihood of its adoption by practitioners and improving its chances of affecting employment outcomes of individuals with disabilities. In a resource-limited environment, evidence of efficacy and effectiveness can help make programmatic funding decisions more rational and, hopefully, more equitable (Brannon, 2010). While RSA is calling on researchers to develop EBPs to enhance VR consumer employment outcomes and in doing so supporting the effectiveness of the VR program, state VR agencies must also rise to the challenge.

\subsection{Evidence-based practices (EBP)}

EBP research has centered on the efficacy of EBPs in the provision of VR service delivery. However, there is acknowledgement of how EBPs influence other areas of rehabilitation counseling practice and service delivery. Current EBP initiatives described in the literature cover a variety of topics ranging from identifying EBP at the state agency level, EBP decision making models, EBP validity, pedagogy, ethics, barriers, facilitators of EBP, and collaboration focused on designing, implementing and adaptation of emerging best practices. A brief review of the empirical literature addresses these ancillary issues influencing EBP and service delivery.

Fleming, Del Valle, Muwoong, and Leahy (2013) conducted a literature review focused on identifying empirical studies of "active VR services" at the state VR agency level published in the last 25 years. "Active services" was defined as actual state VR agency service delivery practices considered "best practices" leading to competitive employment outcomes for individuals with disabilities receiving VR services through the state VR agency. The best practices that emerged from the literature were; interagency collaboration, counselor education and consumer outcomes, services to a targeted group, EBP supported employment, empowerment and consumer self-concept, essential elements of service delivery, and miscellaneous VR services and outcomes.

Although existing in specific areas of rehabilitation counseling and VR services delivery such as EBP supported employment, EBPs are still not a common practice at the system or practitioner level, are inconsistent in application and scope, and lack a formal methodological approach on designing, implementing and analyzing results. While not as well developed as other disciplines (i.e. psychiatric rehabilitation), the literature does reflect attempts to develop and apply EBP at the system and practitioner levels. These efforts are found within several areas such as interagency collaborations and supported employment services being the most widely referenced best practices in the field.

Strauser and Wong (2010) contend EBP research that focuses primarily on intervention efficacy is theoretically flawed by not addressing the construct of external validity. They suggest implementing the REAIM framework. This framework is grounded in 
systems-based and social-ecological theory with the central tenet being the ultimate impact of an intervention is due to the combination and interaction of the following five evaluative dimensions: Reach, Efficacy (Effectiveness), Adoption, Implementation, and Maintenance (Glasgow, McKay, Piette, \& Reynolds, 2001; Glasgow, Vogt, \& Boles, 1999). The RE-AIM model provides a method by which to evaluate rehabilitation interventions and programs. The RE-AIM evaluation model emphasizes the reach and representation of both participants and settings by evaluating the impact on interventions, programs, and policies over five dimensions including intervention efficacy.

Tarvydas, Addy, and Fleming (2010) provide a new perspective of EBP research in rehabilitation counseling as moving from a dichotomy for "either/or" to a dialectic of "this/and" thinking. This approach allows for the inclusion of critical aspects of EBP to the longstanding practices in rehabilitation counseling research and practice. Tarvydas et al. (2010) contend that EBP upholds ethical principles by allowing consumers to make informed choices and supports the five major ethical principles of autonomy, justice, fidelity, beneficence and non-maleficence. This includes support for the CRCC Code of Professional Ethics (CRCC, 2010). Specifically for Standard D.1-Commpetency to provide competent services to their clients and not to provide services not personally competent to render and Standard D.6.a in which rehabilitation counselors are obligated to use techniques and procedures that have an empirical or scientific foundation.

Chan et al. (2010) cited multiple challenges to EBP implementation including: a) the broad array of services provided within the rehabilitation counseling scope of practice, b) the lack of scientific rigor in rehabilitation counseling research, c) rehabilitation counselors may not possess the skills necessary to evaluate and incorporate research findings into practice, d) limited training in academic search skills, and e) time and potential organizational barriers. Graham et al. (2013) conducted a study to identify barriers and facilitators to the use of EBP by professional staff of state VR agencies. The results indicate that the majority of VR staff who participated in the study value research for practice. However, multiple barriers limit EBP implementation. The barriers include the findings that EBP is not widely encouraged by the agency, rehabilitation counselors are not expected to use EBP in service provision, lack of agency resources, limited counselor time to research EBP, and lack of agency incentives to incorporate EBP into service provision. Graham et al. (2013) noted the consistently high unemployment rate for individuals with disabilities. The authors stress the importance of EBP in VR service delivery as a bridge for unemployed individuals with disabilities to gain employment.

As stated earlier, the realm of EBP has moved beyond demonstrating efficacy of EBP interventions. EBP is now viewed as having influence across a wide spectrum of rehabilitation counseling practices and VR service delivery practices by identifying EBPs at the state agency level, EBP decision making models, EBP validity, pedagogy, ethics, barriers and facilitators of EBP and collaboration focused on designing, implementing and adaptation of emerging best practices. As the movement towards EBP in rehabilitation counseling continues, it will most likely include other areas within rehabilitation counseling and VR service delivery yet discovered. This will provide opportunities for further research of into the breath and complexities of implementing EBP in rehabilitation counseling and VR service delivery.

\section{Methodology}

This study utilized a modified version of the consensual qualitative research (CQR) methodology developed by Hill and associates to analyze data within a multiple case study design (Hill, Thompson, \& Williams, 1997; Hill \& Williams, 2012). The modified CQR process involved key techniques central to qualitative research including semi-structured questions that were used to gather data and study the cases as well as a comprehensive review of administrative reports. Semi-structured interview methods are frequently used in qualitative research and provide an open-ended and flexible manner of gathering information from participants (Merriam, 2009). Consistent research procedures were used across the four state vocational rehabilitation program sites to ensure representativeness in regard to data collected, analysis, and interpretation of the findings (Stake, 2006; Yin, 2009).

The research question, "What are the specific best practices that appear to be evidence-based and transportable to other state VR agencies?" was specifically proposed to identify innovative, emerging, promising, or best practices in the public vocational rehabilitation program. Case studies explore bounded, or defined, systems through in-depth data collection that involve multiple sources of information and result in a rich description of themes (Creswell, 2007; Stake, 1995). The research question was designed to identify and 
gather information regarding services and practices believed to be evidence-based, innovative, or promising and contribute to improved consumer employment outcomes specifically within the state-federal vocational rehabilitation program.

\subsection{Participants}

A multiple case study analysis was designed to explore best practice models of effective VR service delivery across four states (Leahy, Del Valle, Fleming, \& Kim, 2012). Participants included administrators, mid-level managers, and counselors from the Maryland, Mississippi, Texas and Utah vocational rehabilitation programs.

Apt representation of staff across the three organizational levels was important in identifying key practices and environmental factors influencing the receptiveness to innovation and adoption of promising or evidence-based practices. Across the four state sites, four directors, 25 Vocational Rehabilitation leaders, 56 mid-level managers, and 73 VR counselors engaged in the study providing perspectives from a total of 158 participants. Researchers made primary contact with agency leaders and these individuals subsequently assumed primary responsibility for participant engagement and coordination efforts. Subjective or purposive sampling (Coyne, 1997; Fraenkel \& Wallen, 2003) is frequently used in qualitative research to select subjects with specified characteristics. In this study, participants were selected based on their role within their organization as well as their experience with innovative or promising practice within the VR program.

Sixty-minute individual interviews were conducted with the respective agency leaders including the state administrator or director as well as the directors of client services and other specific units. Ninety-minute focus groups were conducted with mid-level managers and involved both regional and district directors. Ninetyminute focus groups involving approximately 10 participants each were also conducted with counselors and comparable staff, including those in training or specialized positions, in each of the four case study states.

\subsection{Data collection}

When conducting case study research, it is important to utilize multiple sources of evidence in order to triangulate data and develop converging themes (Yin, 2009). The primary sources of data collected and analyzed in this study involved interviews and focus groups, admin- istrative documents provided by each state's central leadership team, and researcher observations.

\subsubsection{Semi-structured interviews and focus groups}

While individual interviews were the primary data collection method used in this study, a variety of data collection techniques including use of focus groups and mixed method approaches are now considered appropriate when used in conjunction with the CQR framework (Chui, Jackson, Liu, \& Hill, 2012; Hill et al., 2005). Semi-structured interview and focus group questions were developed following guidelines set forth by McCracken (1988) and Morgan (1997). The interviews and focus groups were digitally recorded and the audio recordings were transcribed for analytical use by the researchers.

\section{Consensual Qualitative Research (CQR) analysis}

Data analysis using a modified CQR method involved three prescribed steps: domain identification, core ideas, and cross-analyses. Domains serve as the starting point in grouping large amounts of information and often include context and specific, recognized interventions (Hill et al., 1997). Following an independent review of the data and subsequent domain identification, researchers collectively shared their draft domains, engaged in rich discussion, and reached consensus regarding the final domains used to accurately portray results. Following domain identification, core ideas were developed for all material within each domain across the study. The research team then met to discuss the suggested core areas, including brief summaries, and revise as needed. Consensus regarding the agreedupon core ideas was necessary before moving to the final step of cross-analyzing the data.

The final step in the CQR process involved a crossanalysis concerning the development of categories to describe consistencies across the core ideas within domains (Hill et al., 2005, 1997). Cross-analysis is more complex than the previous steps of domain and core idea identification and allows for a higher level of abstraction. The cross-analysis process required the researchers to derive categories by identifying common themes or elements across responses within the sample. This was a particularly useful analytical exercise with this study given the large amount of data present as well as the multi-level organizational sampling method involved. 


\subsection{Auditor}

Assistance of an external auditor is a unique feature of CQR methodology (Ladany, Thompson, \& Hill, 2012). The auditor reviews the raw data and poses objective recommendations regarding the researchers' adherence to original source data to ensure representativeness and trustworthiness (Schlosser, Dewey, \& Hill, 2012). The auditor for this study was given access to raw data including interview and focus group transcripts and provided objective input to determine whether the data was accurately assigned to the domains and abstracted into core ideas and importantly, reflected the raw data (Hill et al., 1997). As noted by Ladany et al. (2012), an external audit of the cross-analysis phase can be particularly helpful in achieving representativeness and ensuring that results accurately reflect the raw data.

\section{Results}

The results from the multiple case study research best reflect promising practices that appear to be sustainable, replicable, and transportable to other state VR agencies. The identified practices emerged from the semi-structured and focus group interviews in two thematic areas; Promising Organizational Practices and Promising Service Delivery Practices. Promising Organizational Practices emerged as systemic, agency wide changes that foster innovative practices. These practices reflect a philosophical or attitudinal change, encouraged creativity and the development of innovative practices at all levels of the agency. Promising Service Delivery Practices facilitate employment outcomes at the service delivery level. These practices affected service delivery at multiple levels within the agency, community rehabilitation organizations, employers and specific disability populations (Leahy et al., 2013). The Promis- ing Organizational Practices will be presented first, then Promising Service Delivery Practices, followed by a discussion describing the sustainability and portability of these practices to other state VR agencies.

\subsection{Promising organizational practices}

The following Promising Organizational Practices reflect agency wide initiatives in a variety of areas including innovative practice development, technology, service delivery charge, partnerships, service delivery practices and training. The selected Promising Organizational Practices demonstrate innovative practices holding promise for sustainability and portability to other state VR agencies are described below. While these practices do not represent the totality of the study results, they offer insight as to the nature and structure of emerging "best practices" at the organizational level. Table 1 offers an overview of the Promising Service Delivery Practices followed by a brief description.

\subsubsection{Incubator unit}

Key to the development of "best practices" is encouragement from the executive leadership to think outside the box and engage in creating innovative service delivery practices. In Texas, the Department of Assistive and Rehabilitative Services (DARS), included the development of "incubator units" where new approaches to service delivery are piloted that may have merit for implementation throughout the agency or in certain areas of the state. "Incubator unit" startups could occur at any level or location within the agency requiring no formal permission to engage in developing innovative services. The only stipulation was to report or share the results across the agency as a method to encourage further innovative developments in other service delivery areas.

The "incubator unit" is an example of introducing promising practices within the agency that has led to

Table 1

Promising organizational practices

\begin{tabular}{llll}
\hline Texas & Utah & Mississippi & Maryland \\
\hline Incubator Unit & Employer Relations & Rapid Response \& Internal Service Delivery & Emerging Leaders \\
Sharepoint & Data Driven - Return on & Business Development Program \& & Organizational Skills Development \\
& Investment & Employment Coordinators & \\
E3 & Organizational Skills & Strong Business Model & \\
& Enhancement & Specialized Coordinators, Counselors \& & \\
& Transformational Agenda & Caseloads & \\
& & Data Driven - AWARE System & \\
& Linking Innovative Networks of Community \\
& Services & \\
\hline
\end{tabular}


positive changes at multiple levels of the agency. First, the encouragement and acceptance of risk taking has improved the morale of agency staff. Staff does not require "permission" and they are no longer anxious taking risks with developing innovative practices for fear of retribution if the practice fails. On the contrary, staff is now motivated to undertake risks to develop and implement innovative practices in an effort to enhance service delivery to both consumers and employers. It is the expectation of staff to "make decisions and be innovative." Leadership is encouraged to "catch people doing something right, to understand the significance of it and to communicate that."

\subsubsection{Share point}

Technology is also considered a critical element in the development of best practices. An important finding was the use of commercially available cloud-based software system called SharePoint. This software is a web-based application that acts as a central site for staff members to post and share ideas around best practices. It allows staff members to share their innovative practices and communicate any challenges and successes with colleagues across the state. The SharePoint software facilitates the dissemination of innovative practice lessons learned so that other staff members can attempt to replicate the practice while making adjustments based on local service delivery needs. Study participants referred to the Share Point site as an opportunity to "brag and steal" the best ideas and innovative practices from around the state. Agency staff takes pride in sharing their success and enjoy the opportunity to post their innovative service delivery practices as well as lessons learned. All staff members have access to Sharepoint and are encourage to replicate programs that might have merit in a different area of the state and to modify successful innovations to meet local conditions.

\subsubsection{Excellent service, every consumer, every time $(E-3)$}

Facilitation of a new consumer service attitude and service delivery was noted among some study participants. It involved a dual consumer approach with consumers with disabilities and employers being the shared focus for consumer service delivery. Inclusive of the dual consumer approach was the belief that each consumer and employer the agency serves be provided with Excellent Service, Every Consumer, Every Time or $\mathbf{E 3}$ for short. Executive leaders strongly endorse this approach to service delivery and consider $\mathbf{E 3}$ to be a standard practice at all levels throughout the agency.

\subsubsection{Employer relations}

Initially funded by the Medicaid Infrastructure Grant (MIG), the Utah State Office of Rehabilitation (USOR) provides funding for this project, which now consists of funding for two full-time staff. The Employer Relations team provides support and education to businesses that express an interest in hiring and retaining people with disabilities. Central to the efforts of Business Relations is the development and maintaining relationships between the agency and the business partners. Employer Relations counted over 100 business partners actively involved in the hiring or expressing willingness to hire individuals with disabilities. To build and maintain relationships with business partners, the two staff regularly schedule individual meetings with partners. These meetings are typically face-to-face although contact via telephone with job openings and opportunities are a regular occurrence. Employer Relations works with The Council of State Administrators of Vocational Rehabilitations (CSAVR) business relations network and The NET (National Employers Team). The national network works closely with the Utah employer network, PWDNET (People With Disabilities Network). The benefits of these workshops, meetings, and trainings can be understood through the following statement, "the employers, the businesses keep coming back. They're impressed with the quality of candidate. They see the results that we've been able to get for them. And even if somebody doesn't get a job the day of the job fair, six months to a year later, we're seeing people are getting jobs with these businesses, because they've been able to talk to them, find out what's really necessary to get a job with the business, and then they're able to go through that process a little more effectively" (Employer Relations, September 12, 2012).

Mississippi Department of Rehabilitation Services (MDRS) employs Employment Coordinators to perform an integral role in developing and managing business relationships, a key component of the agency's VR Business Development Program. Employment Coordinators work directly with employers to promote MDRS services and share job leads with MDRS staff. The Business Development Program is consistent with the CSAVR "dual consumer" approach, which encourages VR agencies to re-define their consumer base as both individuals with disabilities as well as employers or the business community.

\subsubsection{Strong business model}

Mississippi MDRS leaders note the three key tenets of their business model, "structure, service, and sales", 
that have made the agency what it is today and contribute to ongoing performance excellence. "These elements help foster an environment grounded by a foundational business framework." MDRS is the structural home of their VR program, and is a distinct department within the Mississippi state system. This status as a separate state department, allows agency leaders the freedom to contact legislators directly regarding procuring the resources necessary to operate their program, "if you don't have the resources, you're not going to do the job." The agency also manages a separate 501(c) 3 non-profit organization called AbilityWorks, Inc. to provide complementary services exclusively to MDRS consumers. Staff members are knowledgeable and focus on service outcomes while providing high quality service to consumers and a commitment to "putting the client first." The sales element allows agency leadership to maintain strong working relationships with legislators, employers, partner agencies, and the public. The agency has an Office of Communications and Constituent Relations (OCCR) whose staff members communicate with an average of five legislators a day. The Mississippi Executive Director promotes the agency's services stating, "constituents have a better understanding of program connectedness and benefits to both the disability and business communities." Agency leaders, mid-level managers, and counselors all noted the ability to promote a consistent message, "we're about putting people with disabilities to work, that's our sole mission." (USOR administration, September 11, 2012)

\subsubsection{Rapid response and internal service delivery}

Mississippi MDRS leaders, managers, and counselors noted a commitment to facilitating movement toward employment as efficiently as possible. In Mississippi, VR cases are typically opened within a matter of days and remain open for approximately 15 months compared with the national average of 24 months among comparable VR agencies (RSA, 2012). When asked about service delivery options, leaders noted difficulty in establishing external service capacity in rural Mississippi. Therefore, MDRS leaders opted to provide the majority of their program's employment services internally rather than subcontracting to vendors. In 2012 , approximately $4 \%$ of the annual budget was used to purchase services through vendors; all other services and supports including benefits counseling, job placement, and on-the-job supports were provided internally through the VR infrastructure. The intent of Mississippi MDRS is to provide a "rapid response" approach to consumer service and save resources by providing services internally whenever possible.

Mississippi MDRS also established AbilityWorks, Inc., a network of 17-community rehabilitation sites located across 10 districts throughout the state to serve individuals with the most significant disabilities. AbilityWorks, Inc. is a 501(c) 3 non-profit entity designed to complement the vocational rehabilitation program by providing vocational assessment and evaluation, job training, work experiences, and integrated community-based employment placement services exclusively to VR agency consumers. Inherent within AbilityWorks is the Linking Innovative Networks of Community Services (LINCS) program. LINCS is an entirely community-based alternative available within the broader AbilityWorks program that provides evaluation, training, and work experience opportunities directly with employers rather than in center-based environments. Participants emphasized that the goals of both AbilityWorks and LINCS are to place consumers in competitive employment. At case closure, $63.7 \%$ of Mississippi MDRS consumers were working 35 or more hours per week in competitive employment compared with the national average among combined VR agencies of $52.1 \%$ (RSA, 2012).

\subsubsection{Specialized coordinators, counselors, and caseloads}

Specialized caseloads specifically designed to serve transition-age youth, supported employment, consumers who are Deaf or hard of hearing, and individuals on the autism spectrum were common approaches across the four states participating in this study. Counselors were provided extensive training in how to best work with these populations and also received agency support in terms of resource development or access to staff with specialized expertise. In Texas, there is an emphasis on serving individuals on the autism spectrum and the agency hired a behavior specialist to support counselors serving this population. The Maryland Division of Rehabilitation Services (DORS) has several unique programs including the Acquired Brain Injury (ABI) program in which consumers continue to receive support through post- employment services following successful case closure and evidence-based supported employment. USOR provides services to ex-offenders through an extensive collaboration involving multiple agencies while Mississippi MDRS focuses on serving specialized populations including youth in transition and deaf-blind consumers through intentionally lower- 
ing caseload sizes and providing staff with access to additional training and supports.

\subsubsection{Data driven}

Several state VR programs invested time and resources in monitoring agency outcomes through internal case management systems such as AWARE, the use of program evaluation to determine return on investment (ROI) or program outcome measurement. AWARE data is used to foster a culture of, "high expectations and pride in their high employment outcome rate" and actively uses data to establish goals and monitor performance. Staff members across all levels of the organization including counselors, managers, and leaders are held accountable for achieving the objectives of the organization. ROI data is used to demonstrate the effectiveness of VR services provided to citizens with disabilities by informing state legislators and other community stakeholders of successful VR outcomes, ensuring funding for future programming.

In Utah, the transformational agenda is used to reorient the VR program toward professionalism, has not been dismissive of the realities of operating a multimillion-dollar project in a changing environment. Program directors indicated that the agency looks at each program in terms of impact on clients and a financial analysis with regard to the cost and benefit of services. USOR utilizes a number of metrics beyond return-on-investment to evaluate the quality, effectiveness, and sustainability of programs. For example, based on feedback from clients and community rehabilitation programs (CRP), the agency developed a milestone payment project which required, "changing payment structure for CRPs providing job placement." Program evaluation incorporates the typical elements used by other agencies (i.e., consumer satisfaction, number of closures with employment outcomes) and also considers new metrics in the evaluation projects and goals. Internally, USOR has developed its own list of high quality indicators for job placements, has surveyed employers regarding their experience with the VR program, reviews case notes, and examines referrals for specialized services as well as the rehabilitation plans developed post-specialized service provision.

\subsubsection{Organizational skills enhancement}

Several state agencies have given consideration to "succession planning" and have developed leadership training programs to prepare selected staff for future leadership roles. Leadership training allows participants to obtain insight into the, "broader perspective of the VR program nationally, statewide and within their part of the agency." The training includes access to executive leadership who serve as "mentors" to training participants allowing them to further develop their leadership skills.

USOR has undertaken a process identified as the Transformational Agenda. The process itself would not necessarily be described as a best practice, but rather the development and promotion of an agency culture that would allow best practices to be adopted and innovation to occur. The Transformational Agenda was initially developed by agency leadership in response to a concern over the loss of institutional knowledge due to retirements of key senior personnel and the subsequent promotion of junior people who were relatively new to the agency. The focus of the process evolved from this initial focus to contemplate impact of policy and process on the capacity of USOR to carry out is essential mission. This included the movement from a case management process orientation to a client centered, holistic approach to services. As one staff member stated, "we became so process-focused that we made the client almost secondary to what we were doing. We actually would make decisions based on our process as opposed to what the client needed and what was most appropriate for them."

Maryland DORS has developed a three level leadership program. The basic level is for new staff to get to know the agency. The second level is called, "Emerging Leaders" and it is for staff that have been there a few years. These participants must be, "doing well in their jobs and have supervisory and regional approval to participate." Emerging leaders is about nine months long with specific training opportunities, such as learning how to speak in public. The third level is called Executive Leadership Institute (ELI). It is for those staff "who have been with the agency for some time, have approvals to participate and are ready to be groomed to take on leadership roles up at the higher levels of the agency."

\subsection{Promising Service Delivery Practices}

Promising Service Delivery Practices enhance service delivery at multiple levels of the agency. These practices include service delivery arrangements with CRPs, other state agencies and employers. The selected Promising Service Delivery Practices described below include a combination of strategies to free up counselor time to engage in core job functions, partner with CRPs for service delivery, address employer needs and 
provide services to specific populations. Again, these practices do not represent the totality of the study results. They offer insight as to the nature and structure of emerging "best practices" at the service delivery level. Table 2 offers an overview of the Promising Service Delivery Practices followed by a brief description.

\subsubsection{Valforce}

One agency implemented a local pilot initiative by contracting with a company called Valforce to outsource some of the non-core functions. Valforce contractors may work with consumers beginning at orientation to provide front-end administrative function such as gathering documentation, assisting the consumer with application and conducting vocational assessments. Once the application is completed and necessary documentation is gathered, Valforce staff hand off the consumer's information to a VR counselor to provide core VR functions, such as eligibility determination. VR staff reported having Valforce staff doing the initial "legwork", they are free to work more closely with eligible consumers and those in service status. The Valforce contractor may provide case management services, such as following up on consumers referred to community rehabilitation programs or assisting with job club. Staff stated this approach of allowing a CRP to manage the non-core administrative tasks has led to an increase in service responsiveness, ability to work with business partners and increase in consumer satisfaction.

\subsubsection{Community Rehabilitation Program (CRP) certification}

Several agencies decided it was in the best interest of consumers if all CRPs met basic staff training and service delivery requirements. As an example, CRP staff must complete job coach training, supported employment training and/or management training depending on their duties. All training is delivered on line. Initially some providers refused to be credentialed and left. However, they were replaced by newer CRPs who completed the training. The agencies reported the level of services has improved since the implementation of the training.

CRP certification is critical to the success of the Maryland DORS evidence-based individualized placement support (IPS) supported employment model. If providers are "not licensed or Commission on Accreditation of Rehabilitation Facilities (CARF) accredited, then the VR agency does a CARF like survey" to accredit the provider. In Maryland, "expectations are set early in the relationship" as fidelity to the IPS model is vital to the success of the program.

\subsubsection{DARSforce}

A promising practice at the state level is the use of a web based cloud technology called DARSforce. DARSforce was launched as a tool to manage business relations and assist staff in their efforts to bring jobready consumers and businesses together. DARSforce enables staff within a division to leverage information regarding existing and potential business relationships and to access information on available embedded training. Additionally, the top 100 businesses with whom the agency partners have a portal to post job openings and access qualified, job ready applicants.

\subsubsection{Embedded Training Programs (ETP)}

ETP programs are promoted as a marketing tool and method to obtain employer buy-in for placing and hiring a consumer. The ETP came about as a result of consumers not having the physical tolerance for work and often being let go by the employer prior to a rehabilitated closure. These programs consist of work conditioning that support the consumer on the job until the productivity and work tolerance are achieved. The ETP is similar to the supported employment "placement and train" model. However, the consumers are

Table 2

Promising Service Delivery Practices

\begin{tabular}{llll}
\hline Texas & Utah & Mississippi & Maryland \\
\hline Valforce & Supported Job Based Training \& Supported & Soft Skills Training & Benefits Counseling \\
CRP Certification & Employment & & $\begin{array}{c}\text { Maryland Seamless Transition } \\
\text { Collaborative }\end{array}$ \\
Chose to Work & Acquired Brain Injury Program \\
Earsforce & Work Incentive Planning Services & Individual Placement \& Support \\
Training & Utah Defendant Offender Workforce & & \\
Programs & Development Taskforce (UDOWD) & & \\
& Career Exploration Services & \\
\hline
\end{tabular}


not supported employment consumers. Participants are placed in an employment setting and trained with supportive services as needed to help the consumer be successful. Once the training is completed, successful participants are hired by the host company or are placed in employment within the same industry. Employers report being satisfied with this program as it relieves them of the burden of training and making work adjustments in order for the consumer to become an employee.

\subsubsection{Choose to Work}

Choose to Work (CTW) is a partnership program between USOR and Utah's Department of Workforce Services. The CTW program is similar to other state agencies' attempts to serve recipients enrolled in the Temporary Assistance to Needy Families (TANF) program who may have substantial barriers to employment due to disabilities. The program is designed to increase employment outcomes for individuals with disabilities who may not need as intensive services as a job coach or supported job-based training (SJBT) but who have been unsuccessful in obtaining employment through traditional efforts.

\subsubsection{Utah Defendant Offender Workforce Development Taskforce (UDOWD)}

Under an American Recovery and Reinvestment Act Justice Assistance Grant, the Utah Department of Corrections (UDC) created a program in collaboration with allied agencies to provide job development and offer direct assistance to individuals under the jurisdiction of UDC. The success of this program lies in the long term, positive partnership between the USOR VR and UDC. A crucial element of this partnership is the long term involvement and commitment of key UDC and USOR personnel to the program and is referred to as the UDOWD Task Force.

The UDOWD Task Force, established to guide the efforts under this program, attempts to implement evidence-based practices in the several ways. First, each ex-offender served by the program receives a thorough assessment with regard to employment goals, inherent risks of certain environments with regarding to recidivism, and the needs of the individual. The program utilizes positive psychology approaches of using job readiness training and employment to bolster intrinsic motivation to remain in the community. After individuals achieve employment, UDOWD also provides on-going support in the community through collaboration and inter-agency cross training.

\subsubsection{Career exploration services}

While career exploration services may be considered a "best practice", this particular service reflects a change in the agency's re-definition of the counselor role, i.e. returning to an emphasis on counseling vs. case management, and engaging the consumer in a more "holistic" manner. USOR's Career Exploration Services (CES) is a unit within the state VR agency that provides vocational evaluation and testing for individuals accepted for services. Part of a more "comprehensive" approach to individual consumer services there are two levels of assessment. Level 1 identifies level of academic achievement, general reasoning ability, vocational interests, and work values. Depending on the client and referral request, Level 2 evaluations may range from 1-5 days in duration and includes instruments focused on achievement testing, aptitude testing, interest inventories, reasoning tests, specific aptitude testing, and work samples. Client interviews and evaluator behavioral observations are also incorporated into Level 2 evaluations as a measure of work tolerance and work behaviors. The results of the evaluation, including observations and interviews, are submitted to the USOR counselor in the form of a comprehensive vocational evaluation report. A recent pilot project between CES and USOR counselors required every referral to be staffed with the evaluator before being scheduling. During these staffing meetings, the rehabilitation counselor, client, and CES evaluator met to discuss the goal of the evaluation and information needed for development of the vocational plan. The result of this project was shorter wait times for evaluation appointments, more streamlined reports, and evaluations that were tailored to individual client needs.

\subsubsection{Work incentive planning and benefits counseling}

An well received promising practice is the development and implementation of a fee for service benefits counseling program for consumers who receive Supplemental Security Incomes (SSI) or Social Security Disability Income (SSDI). In Maryland, the Benefits Counseling service is based on a partnership with the Maryland Department of Disabilities, who administer the Medicaid Infrastructure Grant (MIG). The agency set up benefits counseling to be a "flexible system that enables clients to return to work with greater confidence." Benefits counseling has helped "to reduce staff fears in working with certain populations." This service is a tool for both counselors and consumers to make more informed decisions about work and how much 
work a client chooses to do. The service has overwhelming support from counselors, with comments from the focus group such as "it's the best thing", "very passionate about it", "it's a life-saver", the service, "helps me manage my caseload and develop better rapport with families", and it has "had an impact on the field." The rehabilitation success rate for SSI/SSDI clients who received benefits counseling was $76.51 \%$ compared to $43.36 \%$ for those consumers who did not receive the service.

USOR operates the Work Incentive Planning Services (UWIPS) program created after receiving funding in the form of grants from the Social Security Administration and the State's Governor's Council. The program is staffed by eight full-time benefits specialists, two other specialists, and a director. USOR VR counselors work in collaboration with Benefits Specialists to identify financial scenarios clients may experience and assist clients and counselors in choosing an appropriate vocational goal. Funding of the program is shared between USOR's federal funds, direct state funds and grant from the Utah Department of Workforce Services, and reimbursement funds from the Social Security Administration related to the Ticket-to-Work program. Consumers that received benefits planning services were almost $15 \%$ more likely to be employed at case closure than USOR clients who did not receive this service. With regard to earnings capacity, individuals who received work incentive planning services earned an average of $\$ 451.59$ more per month than those individuals who did not receive this service

\subsubsection{Soft skills training}

Mississippi MDRS staff recognized that many of the individuals they serve lacked critical soft skills such as effective communication, good attendance and punctuality, time management, appropriate grooming and dressing, and the interpersonal interactions with co-workers and supervisors necessary to maintain employment. The agency purchased a soft skills training program called Smart Work Ethics (SWE) that contains a standardized curriculum designed to change behavior and improve employability through an interactive training approach. As of September 2012, approximately 300 consumers had participated in the program. Each participant receives a certificate upon completion of the course and a Smart Work Ethics report is included in each participant's case file. Consistent with their internal service delivery model, Mississippi MDRS integrated the soft skills training into their internal counselor-trainer model. If a coun- selor or staff member is interested in becoming a Smart Work Ethics trainer, they have the option to apply and receive instruction and mentoring internally through the MDRS training cadre. One counselor noted, "I have taught four classes so far ... each class had eight [participants], and I have four or five that already found a job after graduating from SWE."

\subsubsection{Maryland Seamless Transition Collaborative (MSTC)}

The Maryland DORS' Seamless Transition Collaborative (MSTC) is a promising practice that is in the last year of a five year grant. MSTC is based on a framework for optimizing the success of youth called "Guideposts for Success", developed by the National Collaborative for Workforce and Disability/Youth (NCWD/Y). The model components align with each Guidepost area. The five Guidepost areas are; 1) Career Preparation and Work-based Learning Experiences-components include Discovery, Individualized Work-based Experience and Individualized Paid Inclusive Employment, 2) Youth Development and Leadership-Self-determination skills instruction and practice, 3) Connecting Activities-Includes Early Vocational Rehabilitation Agency Case Initiation and System Linkages and Collaboration, 4) Family Involvement and Supports-Includes Family Supports and 5) School-based Preparatory Experiences-Coordination with Teachers and Instructional Staff.

Maryland DORS has partnered with a non-profit organization called TransCen, Inc. to assist with administration of MSTC as part of a RSA demonstration grant. The "goal was inter-agency collaboration to result in sequential delivery of transition services. Students are to have at least one paid work experience before they leave high school." The MSTC "model is designed to be applicable across disability and across students, regardless of the types of special education services or exit documents they receive." It was noted that, "64\% of MSTC participants who exited did so with a job and/or enrollment in post-secondary education, which is much higher than the norm." An important value that frames the model is that, "everybody can work."

\subsubsection{Acquired Brain Injury (ABI) program}

ABI emerged from "collaboration with other providers and other professionals across the state in order to develop a supported employment (SE) program for individuals with acquired brain injury." When ABI clients reach a "minimum 90 days of employment, and 
employment is stable, then the VR agency closes their case." Following case closure, "the same day, the individual's case is moved into post-employment services." The use of post- employment services has resulted in ABI program participants retaining employment for longer periods, and are less likely to return for services through opening a new case.

\subsubsection{Individual Placement and Support (IPS)}

The IPS model is a collaboration between the Maryland DORS agency and the state mental health administration. The IPS model serves individuals with significant mental health issues following the evidencebased supported employment Dartmouth model. The program uses "braided" funding between the state VR and state mental health administration to provide blended services to VR and mental health agency consumers. Unique to this program is a single point of entry and anyone who is eligible for services with the state Mental Hygiene Authority (MHA) is presumed eligible for VR services. A VR application is completed with an Individualized Plan for Employment (IPE) developed within two weeks of application. This rapid response maintains fidelity with the IPS model by facilitating consumer choice and seeks employment based on the consumer's expressed interest in work without delays in service due to policy restrictions. With this model, outcome data has shown the "correlation of $95 \%$ for people that achieve a 45 day placement to ultimately achieve a 90 day placement."

State-federal VR agencies are under pressure to develop evidence-based practices that lead to competitive employment outcomes for people with disabilities and justify continuing program support. The findings from this multiple case study describe promising organizational and service delivery practices that encourage creativity and the development of innovative practices at all levels of the agency. These innovative, best practices were noted in the form of promising organizational practices and promising service delivery practices. Promising Organizational Practices were described as systemic, agency wide changes that foster innovative practice, while Promising Service Delivery Practices facilitate employment outcomes at the service delivery level. The four state VR agency results demonstrate commitment to service delivery improvement through leadership strategies that foster innovation at all levels. This includes promoting an environment in which thinking, planning and implementing creative best practices is to be encouraged and recognized.

\section{Discussion}

The findings from the multiple case study analysis provided the beginnings of understandings of the specific best practices implemented by state VR agencies to serve persons with disabilities seeking employment. The researchers identified 29 agency practices, subdivided into Promising Organizational Practices (15 practices) and Promising Service Delivery Practices (14 practices), that were provided in varying degrees within the state VR agencies involved in this study.

The Promising Organizational Practices are in many ways a direct response to the agency culture and leadership direction within those agencies. That is, absent the agency cultures described by Sherman et al. (2014), the creativity associated with the development and implementation of these practices may not have been realized. Further, as is evident in the Promising Organizational Practices, the drive toward creating a climate that promotes inquiry, innovation, evaluation, and dissemination is consistent with the effort to promote knowledge translation of the experiential knowledge of the leaders, managers, and counselors within state agencies. Practices such as placing an emphasis on skill enhancement, specialize service delivery, and service provision (E-3) demonstrate that agencies are focused on providing clients with the services that can be tailored to meet their needs and that their satisfaction is of the highest importance. Likewise, Promising Organizational Practices identify the value in working with employers (Employer Relations) or at the very least conceptualizing a business model can facilitate a common understanding of the experience of operating a business and considering options to improve that business. Lastly, Promising Organizational Practices identify the need for informed decision-making. Becoming data driven beyond the stipulated federal requirements, sharing and discussing data and interventions among staff at all levels (Sharepoint), and tasking a specific group (Incubator Unit) or the agency as a whole to be innovative and take risks in experimenting with new methods provide the impetus for agencies to take steps in new directions.

As Promising Organizational Practices provide a framework for knowledge translation within the agency, Promise Service Delivery Practices can be seen as the result. The Knowledge-to-Action (KTA) process acknowledges the importance of the local context, in this case specific state VR agency needs, and the need to be flexible and adaptive to sustain efforts to learn from actions and revise those efforts based on out- 
comes (Sudsawad, 2007). The KTA process relies on the exchange between knowledge creation and action to develop and revise services. As a result, the agencies involved in this study each developed practices that met the needs of their respective clients and the specific situational needs of the agency. The result is a focus on improving service delivery at multiple levels within the agency and model these efforts for other providers, employers, and to clients and their families. Maximizing efficiencies through change the methods of service provision (Valforce, Career Exploration Services, Work Incentives Planning, MSTC, ABI program) remove the expectation that every rehabilitation counselor must provide every services and promote the utilization of specialized services that best meet client needs. Through partnering with other agencies to share the responsibility of service provision and outcomes (Choose to Work, UDOWD), state VR agencies can maximize supports for individuals and increase the opportunities for goal attainment. Further, recognizing that the best approach may be directly affecting change in clients and providing them with the skills to succeed (IPS, Soft skills training) stems from the attempts to integrate existing research and provide another facet of services.

\subsection{Sustainability}

In reviewing the efforts of state VR agencies to promote Promising Organizational Practices that foster Promising Service Delivery Practices, the sustainability of these efforts must be evaluated to insure their continuance into the future (Canadian Institutes of Health Research [CIHR], 2012). The agencies involved in this study commented on their sustainability efforts during the course of the interviews. However, the bulk of the comments centered on the cost aspect of ongoing provision of services. Although demonstrating financial viability of services is critical to the capacity of state VR agencies to continue offering them, this may not be the most important consideration in relation to sustainability. Despite efforts to demonstrate the success of the various practices, Organizational or Service Delivery, many agencies have yet to complete an intensive evaluation of these services. Specifically, agencies were able to provide broad findings of the return-on-investment and other salient factors in determining the worth of different practices. However, less clear were the specific conditions that these services achieved maximum benefits with highest efficiency. Understanding which clients, with which specific needs, and when to provide these services was more a function of oral tradition than evidence-based practice. Without this knowledge, the ability to continue to be flexible and adaptive, to make informed decisions, and to continue to offer these service is at risk (CIHR). Likewise, as certain services grew out of innovations to address local, regional, or statespecific concerns, additional investigation into the properties of these practices is needed to effectively translate and transport these services into other agencies.

\subsection{Portability}

State VR agencies that have implemented the Promising Organizational Practices and the Promising Service Delivery Practices described in this report expressed satisfaction with these practices and perceive value in their development and maintenance. However, transporting and replicating these services to other state VR agencies requires several considerations. First, the portability of services should be considered a function of how success is defined and the evidence required to evaluate if that successful outcome has occurred (Probst, 2008). Each state VR agency contemplating adopting the practices developed in other states must conceptualize the outcome they are trying to achieve as a function of offering a certain service. In addition, state VR agencies should consider defining the targeted outcome beyond the identifying those individuals employed at closure. Rather, outcomes should reflect theory-driven, evidence-based factors that are associated with leading to or supportive a range of rehabilitation outcomes. Developing a clear concept of that outcome will also support the selection of evaluation methods to assess progress. Adopting measurement standards, standards based on proven assessments, will allow state VR agencies to evaluate the efficacy of practices. Through careful selection of measures, agencies can identify potential limitations in their delivery, assess progress toward the stated outcome, and adjust the delivery of the practice to maximize benefit for the consumer. However, the most important factor in determining the portability of promising practices may lie in the culture of the agency. Agency culture provides the impetus that drives agencies to identify service needs, review practices that best fit those needs, and implement those services that provide the greatest benefits.

\subsection{Implications}

Knowledge translation, such as the KTA process, relies on the inputs from actions. This input informs 
research and provides a mechanism to further the stateof-the-science. However, the process is only as good as the information being transferred within the process. Anecdotal reports, RSA 911 data, and economic viability plans, while valuable, do not provide the level of detail needed for the articulation of an evidence-based practice. Subsequently, efforts to translate knowledge from one agency to another is problematic as concerns will undoubtedly arise related to the utility of the services varying from one state VR agencies to another. As these variable outcomes are identified, the natural convention is to assume that a process may not have the assumed efficacy and be discontinued on the basis of this assumption.

To promote the adoption and translation of services, researchers, state VR agencies, and other stakeholders must work together to establish a basic framework to evaluate emerging ideas or promising practices (CIHR, 2012). Knowledge translation, at least as it is theorized in the KTA process, is a cyclical progression requiring input from all stakeholders throughout. By working together to identify such tools such as basic measurement components, active data collection and analysis, manualization of practices, and fidelity assessments of practitioners providing an intervention, the capacity to identify evidence-based practices and the specific conditions required for those practices becomes possible (Sudsawad, 2007). Chan et al. (2010) provide a four step clinical decision-making model to assist with implementing EBPs. These steps are; 1) formulate well defined, answerable question(s), 2) seek best evidence to answer the question(s), 3) critically appraise the evidence and 4) apply evidence to the individual client. However, the first step in such an undertaking may be the adoption of the cultural practices (Leahy et al., 2013) described in this report and reviewed in detail by Sherman et al. (2014).

A collaborative undertaking such as this can be model on existing frameworks. For example, Greene and Kenney (2013) describe a collaborative project between the Southeast Training Assistance \& Continuing Education Center (TACE) Region IV, four state VR agencies (Florida DVR, Mississippi DRS, Georgia GVRA, Kentucky OVR) and CRPs to integrate innovative practices within the state VR service delivery mainstream. Southeast TACE assists states with introducing or integrating effective practices into their service portfolios and proposes projects that will meet organization needs and lead to improved performance and outcomes (Greene \& Kenney, 2013). TACE works with the state VR agencies and CRPs to identify a practice, learn it and embed it in policy and operations. Each project takes a phased in approach, starting with a pilot test and progressing to replication and dissemination. The projects have had impact in the following areas; 1) increased awareness, understanding and utilization among state VR leaders and partners and 2) infrastructure-policy, regulations, capacity building and partnerships leading to policy changes, internal/external capacity increases and stronger partnerships. By extending collaboration in projects such as this to include the methodological rigor necessary to determine evidence-based practices, advancing the state-of-the-science may be closer than might be believed.

\section{Acknowledgments}

The contents of this article were developed with support from the Rehabilitation Research and Training Center on Evidence-Based Practice in Vocational Rehabilitation (RRTC-EBP VR) at the University of Wisconsin-Madison and the University of WisconsinStout and with funding provided by the U.S. Department of Education National Institute on Disability and Rehabilitation Research (Grant H133B100034). The ideas, opinions, and conclusions expressed, however, are those of the authors and do not represent recommendations, endorsements, or policies of the U.S. Department of Education.

\section{References}

Brannon, R. (2010). EBP Issue: Forward. Rehabilitation Education, 24(3\&4), 177-178.

Canadian Institutes of Health Research (CIHR) (2012). Guide to knowledge translation planning at CIHR: Integrated and end-of-grant approaches. Retrieved June 16, 2014, from http://www.cihr-irsc.gc.ca/e/45321.html

Chan, F., Bezyak, J., Romero Ramirez, M., Chiu, C., Sung, C., \& Fujikawa, M. (2010). Concepts, challenges, barriers, and opportunities related to evidence-based practice in rehabilitation counseling. Rehabilitation Education, 24(3\&4), 179-190.

Chan, F., Rosenthal, D. A., \& Pruett, S. (2008). Evidence-based practices in the provision of rehabilitation services. Journal of Rehabilitation, 74(2), 1-5.

Chan, F., Tarvydas, V., Blalock, K., Strauser, D., \& Atkins, B. J. (2009). Unifying and elevating rehabilitation counseling through model-driven, diversity-sensitive evidence-based practice. Rehabilitation Counseling Bulletin, 52, 114-119.

Chui, H. T., Jackson, J. L., Liu, J., \& Hill, C. E. (2012). Annotated bibliography of studies using consensual qualitative research. In C. E. Hill (Ed.), Consensual qualitative research: A practical resource for investigating social science phenomena (pp. 213-266). Washington, DC: American Psychological Association. 
Commission on Rehabilitation Counselor Certification (CRCC) (2010). Code of Professional Ethics for Rehabilitation Counselors. Schaumburg, IL: CRCC.

Coyne, I. T. (1997). Sampling in qualitative research. Purposeful and theoretical sampling; merging or clear boundaries? Journal of Advanced Nursing, 26, 623-630.

Creswell, J. W. (2007). Qualitative inquiry \& research design: Choosing among five approaches. (2nd ed.). Thousand Oaks, CA: Sage.

Fleming, A. R., Del Valle, R., Muwoong, K., \& Leahy, M. J. (2013). Best practice models of effective vocational rehabilitation service delivery in the public rehabilitation program: A review and synthesis of the empirical literature. Rehabilitation Counseling Bulletin, 56(3), 146-159.

Fraenkel, J. R., \& Wallen, N. E. (2003). How to design and evaluate research in education. New York, NY: McGraw-Hill.

Glasgow, R. E., McKay, H. G., Piette, J. D., \& Reynolds, K. D. (2001). The RE-AIM framework for evaluating interventions: What can it tell us about approaches to chronic illness management? Patient Education and Counseling, 44, 119-127.

Glasgow, R. E., Vogt, T. M., \& Boles, S. M. (1999). Evaluating the public health impact of health promotion interventions: The RE-AIM framework. American Journal of Public Health, 89, 1322-1327.

Graham, C., Inge, K., Wehman, P., Murphy, K., Revell, W. G., \& West, M. (2013). Moving employment research into practice: Knowledge and application of evidence-based practices by state vocational rehabilitation agency staff. Journal of Vocational Rehabilitation, 39, 75-81.

Greene, D. L., \& Kenney, C. (2013). Southeast TACE region IV impact of innovations projects on VR adoption of emerging practices 2009-2012. Journal of Rehabilitation Administration, 37(1), $35-42$.

Hill, C. E., Knox, S., Thompson, B. J., Williams, E. N., Hess, S. A. \& Ladany, N. (2005). Consensual qualitative research: An update. Journal of Counseling Psychology, 52, 196-205.

Hill, C. E., Thompson, B. J., \& Williams, E. N. (1997). A guide to conducting consensual qualitative research. The Counseling Psychologist, 25, 517-572.

Hill, C. E., \& Williams, E. N. (2012). The sample. In C. E. Hill (Ed.), Consensual qualitative research: A practical resource for investigating social science phenomena (pp. 71-82). Washington, D.C.: American Psychological Association.

Koscuilek, J. F. (2004). Research applications in the longitudinal study of the vocational rehabilitation services program. Rehabilitation Counseling Bulletin, 47, 173.

Ladany, N., Thompson, B. J., \& Hill, C. E. (2012). Crossanalysis. In C. E. Hill (Ed.), Consensual qualitative research: A practical resource for investigating social science phenomena (pp. 117-134). Washington, DC: American Psychological Association.

Law, M. (2002). Evidence-based rehabilitation: A guide to practice. Thorofare, NJ: SLACK.

Leahy, M. J., \& Arokiasamy, C. V. (2010). Prologue: Evidence-based practice research and knowledge translation in rehabilitation counseling. Rehabilitation Education, 24(3\&4), 173176.

Leahy, M. J., Del Valle, R., Fleming, A. R., \& Kim, M. (2012). Research protocol: Multiple case studies on effective vocational rehabilitation service delivery practices. Lansing, MI: Rehabilitation Research and Training Center on Effective VR Service Delivery Practices.
Leahy, M. J., Del Valle, R. J., Sherman, S. G., Anderson, C., Tansey, T., Lui, J., \& Fitzgerald, S. (2013). Phase II final report: Multiple case studies on effective vocational rehabilitation services delivery practices. Lansing, MI: Rehabilitation Research and Training Center on Effective VR Service Delivery Practices.

Leahy, M. J., Thielsen, V. T., Millington, M. J., Austin, B., \& Fleming, A. (2009). Quality assurance and program evaluation: Terms, models, and applications in rehabilitation administration. Journal of Rehabilitation Administration, 33(2), 69-82.

McCracken, G. (1988). The long interview. Newbury Park, CA: Sage.

Merriam, S. B. (2009). Qualitative research: A guide to design and implementation. San Francisco, CA: Jossey-Bass.

Morgan, D. L. (1997). Focus groups as qualitative research (2nd ed.). Thousand Oaks, CA: Sage.

Patton, M. Q. (2008). Utilization-Focused Evaluation (4thed.). Thousand Oaks, CA: Sage.

Paul, L. (1967). Strategy of outcome research in psychotherapy. Journal of Counseling Psychology (31), 109-118.

Probst, B. (2008). Issues in portability of evidence-based treatment for adolescent depression. Child Adolescent Social Work Journal, 25, 111-123.

Pruett, S. R., Rosenthal, D. A., Swett, E. A., Lee, G. K., \& Chan, F. (2008). Empirical evidence supporting the effectiveness of vocational rehabilitation. Journal of Rehabilitation, 74, 56-63.

Rubin, S. E., Chan, R., \& Thomas, D. (2003). Assessing changes in life skills and quality of life resulting from rehabilitation services. Journal of Rehabilitation, 69, 4-9.

Schlosser, L. Z., Dewey, J. H., \& Hill, C. E. (2012). Auditing. In C. E. Hill (Ed.), Consensual qualitative research: A practical resource for investigating social science phenomena (pp. 135-144). Washington, DC: American Psychological Association.

Sherman, S., Leahy, M. J., Del Valle, R., Anderson, C. A., Tansey, T. N., \& Lui, K. (2014). Organizational and cultural factors that promote creative best practices in the public rehabilitation program: Findings from a four-state multiple case study. Journal of Vocational Rehabilitation, 41(2), 115-125.

Stake, R. E. (1995). The art of case study research. Thousand Oaks, CA: Sage.

Stake, R. E. (2006). Multiple case study analysis. New York, NY: Guilford Press.

Strauser, D. R., \& Wong, A. W. (2010). Impact not efficacy: Applying the RE-AIM framework to rehabilitation counseling research. Rehabilitation Education, 24(3\&4), 213-224.

Sudsawad, P. (2007). Knowledge translation: Introduction to models, strategies, and measures. Austin, TX: Southwest Educational Development Laboratory, National Center for the Dissemination of Disability Research.

Tarvydas, V., Addy, A., \& Fleming, A. (2010). Reconciling evidencebased research practice with rehabilitation philosophy, ethics and practice: From dichotomy to dialectic. Rehabilitation Education, 24(3\&4), 191-204.

U.S. Department of Education, Office of Special Education and Rehabilitative Services, Rehabilitation Services Administration (RSA) (2012). State plan for the state vocational rehabilitation services program and state plan supplement for the state supported employment services program: Mississippi department of rehabilitation services. Retrieved from http:// rsa.ed.gov/view.cfm?rsaform $=V R \% 20$ State $\% 20$ Plan\&state $=$ MS \&agency $=\mathrm{C} \& \mathrm{fy}=2012$

Yin, R. K. (2009). Case study research: Design and methods (4th ed.). Washington, DC: Sage. 